\title{
Estratégias utilizadas na atenção primária para inclusão do homem nas ações de saúde: uma revisão integrativa
}

\author{
Strategies used in primary care to include men in health actions: an integrative review
}

Estrategias utilizadas en atención primaria para incluir al hombre en las acciones de salud: una revisión integradora

\begin{abstract}
RESUMO
Objetivo: Identificar as estratégias utilizadas na atenção primária para a inclusão do homem nas ações de saúde. Método: Trata-se de uma revisão integrativa da literatura realizada a partir das bases de dados SciELO, LILACS e BDENF entre os meses de setembro e outubro de 2020 as quais geraram 184 referências. Após remoção das duplicatas e emprego dos critérios de inclusão e exclusão a amostra final foi composta por 15 artigos. Resultados: Identificou-se como estratégias para inclusão do homem a visita domiciliar, a realização de práticas dialogadas por meio de palestras e campanhas educativas, além do atendimento noturno. Dentre as fragilidades, destacam-se a ausência de ações promoção e prevenção da saúde, o déficit de investimento, insumos e infraestrutura e o desconhecimento da PNAISH. Conclusão: Ações direcionadas à saúde do homem é primordial para estimular que estes compreendam suas necessidades de saúde, bem como adote medidas preventivas.
\end{abstract}

DESCRITORES: Saúde do homem; Atenção primária à saúde; Estratégia saúde da família; Acesso aos serviços de saúde.

\section{ABSTRACT}

Objective: To identify the strategies used in primary care for the inclusion of men in health actions. Method: This is an integrative literature review carried out from the SciELO, LILACS and BDENF databases between the months of September and October 2020, which generated 184 references. After removing duplicates and using the inclusion and exclusion criteria, the final sample consisted of 15 articles. Results: It was identified as strategies for the inclusion of men the home visit, the carrying out of practices dialogued through lectures and educational campaigns, in addition to night care. Among the weaknesses, the absence of health promotion and prevention actions, the lack of investment, inputs and infrastructure and the lack of knowledge of PNAISH stand out. Conclusion: Actions aimed at men's health are essential to encourage them to understand their health needs, as well as adopt preventive measures.

DESCRIPTORS: Men's health; Primary health care; Family health strategy; Health services accesibility.

\section{RESUMEN}

Objetivo: Identificar las estrategias utilizadas en atención primaria para la inclusión del hombre en las acciones de salud. Método: Se trata de una revisión integrativa de la literatura realizada a partir de las bases de datos SciELO, LILACS y BDENF entre los meses de septiembre y octubre de 2020, que generó 184 referencias. Después de eliminar los duplicados y utilizar los criterios de inclusión y exclusión, la muestra final fue de 15 artículos. Resultados: Se identificó como estrategias para la inclusión de los hombres la visita domiciliaria, la realización de prácticas dialogadas a través de charlas y campañas educativas, además del cuidado nocturno. Entre las debilidades destacan la ausencia de acciones de promoción y prevención de la salud, la falta de inversión, insumos e infraestructura y el desconocimiento del PNAISH. Conclusión: Las acciones dirigidas a la salud de los hombres son fundamentales para que comprendan sus necesidades de salud y adopten medidas preventivas.

DESCRIPTORES: Salud del hombre; Atención primaria de salud; Estrategia de salud familiar; Acessibilidad a los servicios de salud.

RECEBIDO EM: 03/04/2021 APROVADO EM: 23/04/2021

\section{Gabriel da Silva Bacelar}

Curso de Graduação em Enfermagem. Universidade Paulista (UNIP), Campus Brasília-DF.

ORCID: 0000-0003-4870-6902

\section{Ricardo Saraiva Aguiar}

Professor Assistente. Curso de Graduação em Enfermagem. Universidade Paulista (UNIP), Campus Brasília-DF.

ORCID: 0000-0003-0335-2194 


\section{INTRODUÇÃO}

$\mathbf{N}$ o Brasil, os princípios e diretrizes da Política Nacional de Atenção Integral à Saúde do Homem (PNAISH) foram publicados em 2008 e regulamentados oficialmente no ano de 2009. Com a formulação da PNAISH, pôde-se nortear as ações que deveriam ser implementadas para se executar o cuidado integral à saúde do homem baseado em suas necessidades para reduzir a sua morbimortalidade e ampliar o seu acesso aos serviços de saúde. ${ }^{1-2}$

A PNAISH visa implantar e/ou estimular nos serviços de saúde, públicos e privados, uma rede de atenção integral à saúde do homem que garanta linhas de cuidado na perspectiva da integralidade capazes de formar e qualificar os profissionais para o seu adequado atendimento. ${ }^{1,3}$

Contudo, os profissionais da Atenção Primária à Saúde (APS) ainda têm conhecimento escasso acerca da PNAISH e os poucos que sabem da sua existência, conhecem com limitações, pois alegam que há pouco incentivo para se trabalhar com o público masculino por parte da gestão, já que na maioria das vezes realizam capacitações para outros públicos e raramente para a população masculina. ${ }^{2}$

Diante disso, a atenção à saúde do homem deve tornar-se parte das ações promovidas pelos serviços APS com a finalidade de proporcionar a ampliação das discussões acerca dessa recente ótica. Incentiva-se assim, por meio da articulação de mecanismos que façam uma correlação entre educação, saúde e promoção da autonomia dos indivíduos em sua escolha por hábitos que possam contribuir para minimizar os riscos e possibilitar que eles vivam de forma mais saudável. ${ }^{4-5}$

Constata-se que apesar do público fe- minino ser maior nos serviços de saúde, os homens são os que mais padecem de agravos e mortalidade causadas por doenças cerebrovasculares, causas externas e cânceres. Ao contrário do que se gostaria, os homens costumam não buscar os serviços de saúde da APS e acabam recorrendo a serviços de alta complexidade quando já não suportam a dor ou quando se tornam sintomáticos de doenças severas. ${ }^{6}$

Assim, percebe-se que por questões culturais, os homens colocam a si mesmo em perigo, quando não buscam em tempo oportuno assistência à saúde. Os homens são estimulados a mostra-se viris e invulneráveis, onde a busca por atendimento na APS poderia defini-los como fracos, medrosos e inseguros, o que ao seu ver os aproximariam da representatividade feminina. Reconhecer essas questões requer estratégias inclusivas para que o acesso dos homens a saúde primária seja facilitado. ${ }^{6}$

Para evitar isso, as Unidades Básicas de Saúde (UBS) vêm adotando estratégias na intenção de incluir o homem nas ações de saúde a partir de um atendimento diferenciado para a população masculina em horário especial noturno, por exemplo., Assim, investigar os fatores que potencializam e fragilizam as estratégias de inclusão do público masculino, pode gerar intervenções que implicarão transformações no processo de acolhimento e fornecerá subsídios para o planejamento de ações voltadas a saúde masculina. ${ }^{8-9}$

Portanto, o objetivo do presente estudo foi identificar as estratégias utilizadas na atenção primária para a inclusão do homem nas ações de saúde. Sob esta perspectiva, destaca-se a pergunta norteadora: quais as estratégias utilizadas pela atenção primária para a inclusão do homem nas ações de saúde?

\section{MÉTODOS}

Trata-se de uma revisão integrativa da literatura realizada em sete etapas ${ }^{10}: 1$ ) delimitação da pergunta norteadora da revisão; 2) definição dos critérios de inclusão e exclusão; 3) busca extensiva da literatura; 4) identificação de potenciais estudos por meio da avaliação do título e resumo; 5) seleção dos artigos com base no texto completo; 6) avaliação da qualidade dos estudos inclusos; 7) síntese dos estudos inclusos.

Tendo em vista a primeira fase da revisão, elaborou-se a pergunta norteadora de pesquisa com base na estratégia PICO: P - população e problema; I - intervenção; C - comparação; e O - outcome (termo em inglês que significa desfecho)11. Assim, considerou-se $\mathrm{P}$ : homens na atenção primária; I: estratégias de acesso; C: qualquer comparação relacionada aos meios utilizados para a inserção do homem nas ações de saúde; O: assistência à saúde. Nesta direção, a pergunta construída foi: quais as estratégias utilizadas pela atenção primária para a inclusão do homem nas ações de saúde?

A busca dos artigos foi realizada entre os meses de setembro e outubro de 2020 nas bases de dados eletrônicas Scientific Electronic Library Online (SciELO), Literatura Científica e Técnica da América Latina e Caribe (LILACS) e Base de Dados em Enfermagem (BDENF).

Para definição dos termos de busca, foi realizada consulta aos Descritores em Ciências da Saúde (DeCS). Elegeu-se o descritor "saúde do homem" que foi combinado com o termo de busca "atenção primária à saúde", "estratégia saúde da família" e "acesso aos serviços de saúde”. Utilizou-se o operador booleano "AND" para combinação. As estratégias construídas

Quadro 1. Estratégias de busca e resultados das produções identificadas. Brasilia, Distrito Federal, 2020.

\begin{tabular}{|l|c|c|}
\hline FONTES DE INFORMAÇÃO & EXPRESSÕES DE BUSCA & RESULTADOS \\
\hline SCIELO & "saúde do homem" AND "atenção primária a saúde" AND "estratégia saúde da família" & 15 \\
\hline LILACS & AND "acesso aos serviços de saúde" & 137 \\
\hline
\end{tabular}




\section{artigo}

Bacelar, G.S.; Aguiar, R.S.

Estratégias utilizadas na atenção primária para inclusão do homem nas ações de saúde: uma revisão integrativa

\begin{tabular}{|l|c|c|}
\hline BDENF & $\begin{array}{c}\text { "saúde do homem" AND "atenção primária a saúde" AND "estratégia saúde da família" } \\
\text { AND "acesso aos serviços de saúde" }\end{array}$ & $\begin{array}{c}32 \\
\text { TOTAL }\end{array}$ \\
\hline \multicolumn{2}{|c|}{184} \\
\hline Elaboração: Bacelar GS, Aguiar RS, 2020.
\end{tabular}

com os termos de busca e seus resultados são apresentados no Quadro 1.

Teve-se como critérios de inclusão para a amostra: artigos publicados de forma online nos últimos 6 anos (2014 a 2020); disponíveis em língua portuguesa e na íntegra; estudos no formato de artigos originais oriundos de produções científicas diversificadas. Como critérios de exclusão, enquadraram-se artigos disponíveis em bases de dados internacionais e exclusivamente em língua estrangeira.

A busca nas bases de dados gerou 184 referências. Destas, 15 estavam no SciELO, 137 na LILACS e 32 artigos na BDENF. Reduziu-se, a partir da aplicação dos filtros de inclusão, o número de ocorrên- cia: 10 artigos por estarem duplicados, 30 artigos por terem tema diverso do objetivo proposto, 20 artigos estavam sem resumo e 40 artigos devido a metodologia. Totalizaram-se 84 artigos submetidos à leitura completa e à aplicação dos critérios de exclusão, gerando-se a rejeição de 70 artigos. Constituiu-se assim a amostra revisada de 14 artigos (Figura 1).

Classificaram-se as evidências dos artigos em seis níveis: Nível I - estudos relacionados à metanálise de múltiplos estudos controlados; Nível II - estudos experimentais individuais; Nível III - estudos quase-experimentais, como o ensaio clínico não randomizado, o grupo único pré e pós-teste, além de séries tem-
Figura 1. Fluxograma da busca nas bases de dados segundo recomendações PRISMA. Brasillia, Distrito Federal, 2020.

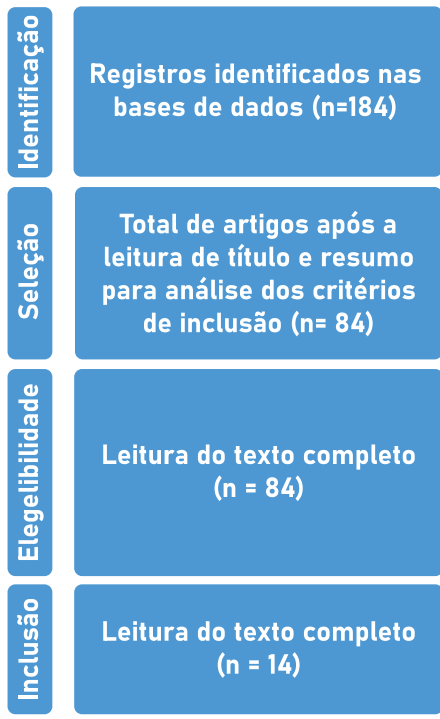

Elaboração: Bacelar GS, Aguiar RS, 2020. porais ou caso-controle; Nível IV - estudos não experimentais, como a pesquisa descritiva, correlacional e comparativa, com abordagem qualitativa e estudos de caso; Nível V - dados de avaliação de programas obtidos de forma sistemática; e Nível VI - opiniões de especialistas, relatos de experiência, consensos, regulamentos e legislações. ${ }^{12}$

Os dados compilados foram então analisados por meio da análise temática ${ }^{13}$, sendo organizados e apresentados em categorias temáticas obtidas a partir das seguintes etapas de análise: 1) familiarização dos dados (resultados dos estudos que compuseram a amostra e se relacionavam com a pergunta da pesquisa); 2) geração de códigos iniciais; 3 ) busca por temas; 4) revisão dos temas; 5) definição e titulação dos temas; 6) produção do relatório.

\section{RESULTADOS}

Elaborou-se, para facilitar a extração e síntese dos dados, uma matriz de síntese descrita em uma planilha de Excel ${ }^{\circ}$. Foram coletados dados como: periódico; país e ano de publicação; autor(es); título; desenho do estudo; principais resultados, fatores relacionados à qualidade da atenção e nível de evidência. Intentou-se com o instrumento, além de formar um banco de dados, mapear pontos pertinentes, integrar dados e caracterizar a amostra revisada. Desse modo, parte destes dados estão representados no Quadro 2.

Grande parcela das publicações refere-se ao ano de 2014 , com quatro artigos (28,6\%), seguidas do ano de 2017 com

Quadro 2. Amostra final de artigos. Brasilia, Distrito Federal, 2020.

\begin{tabular}{|c|c|c|c|c|c|c|}
\hline ESTUDO & PERIÓDICO & AUTOR(ES) & ANO & TítULO & $\begin{array}{c}\text { DESENHO DO } \\
\text { ESTUDO }\end{array}$ & $\begin{array}{c}\text { NIVEL DE } \\
\text { EVIDÊNCIA }\end{array}$ \\
\hline E1 & $\begin{array}{c}\text { Revista Brasileira de } \\
\text { Epidemiologia }\end{array}$ & Alves AN et al. ${ }^{4}$ & 2019 & $\begin{array}{c}\text { Acesso de primeiro contato na atenção } \\
\text { primária: uma avaliação pela população } \\
\text { masculina }\end{array}$ & $\begin{array}{c}\text { Estudo } \\
\text { transversal }\end{array}$ \\
\hline
\end{tabular}




\begin{tabular}{|c|c|c|c|c|c|c|}
\hline E2 & $\begin{array}{l}\text { Revista Baiana de } \\
\text { Enfermagem }\end{array}$ & $\begin{array}{l}\text { Batista BD et } \\
{\text { al. }{ }^{5}}^{5}\end{array}$ & 2019 & $\begin{array}{l}\text { Discurso de homens sobre o acesso à } \\
\text { saúde na atenção básica }\end{array}$ & $\begin{array}{l}\text { Estudo } \\
\text { descritivo }\end{array}$ & IV \\
\hline E3 & $\begin{array}{l}\text { Revista de } \\
\text { Enfermagem UFPE } \\
\text { online }\end{array}$ & $\begin{array}{l}\text { Bacelar AYS et } \\
\text { al. }^{14}\end{array}$ & 2018 & Homens na unidade de saúde da família & Qualitativo & IV \\
\hline E4 & $\begin{array}{c}\text { Revista de } \\
\text { Enfermagem UFPE } \\
\text { online }\end{array}$ & $\begin{array}{l}\text { Barbosa YO et } \\
\text { al. }^{15}\end{array}$ & 2018 & $\begin{array}{l}\text { Acesso dos homens aos serviços de } \\
\text { atenção primária à saúde }\end{array}$ & Quantitativo & IV \\
\hline E5 & $\begin{array}{l}\text { Revista Brasileira de } \\
\text { Enfermagem }\end{array}$ & Silva AN et al. ${ }^{16}$ & 2017 & $\begin{array}{l}\text { A avaliação da atenção primária a saúde } \\
\text { na perspectiva da população masculina }\end{array}$ & $\begin{array}{l}\text { Estudo } \\
\text { transversal }\end{array}$ & IV \\
\hline E6 & Revista de APS & $\begin{array}{l}\text { Martins AM, } \\
\text { Modena }{ }^{\mathrm{CM} 17}\end{array}$ & 2017 & $\begin{array}{c}\text { Acesso da população masculina e } \\
\text { utilização dos serviços de atenção } \\
\text { primária à saúde em belo horizonte - MG }\end{array}$ & $\begin{array}{l}\text { Estudo } \\
\text { exploratório }\end{array}$ & IV \\
\hline E7 & $\begin{array}{l}\text { Revista Cubana de } \\
\text { Enfermería }\end{array}$ & Daher DV et al. ${ }^{1}$ & 2017 & $\begin{array}{l}\text { A construção do vinculo entre o homem } \\
\text { e o serviço de atenção básica de saúde }\end{array}$ & Qualitativo & IV \\
\hline E8 & $\begin{array}{l}\text { Cadernos de Saúde } \\
\text { Pública }\end{array}$ & $\begin{array}{l}\text { Moreira MCN, } \\
\text { Gomes R, } \\
\text { Ribeiro CR }{ }^{18} \\
\end{array}$ & 2016 & $\begin{array}{l}\text { E agora o homem vem? Estratégias de } \\
\text { atenção à saúde dos homens }\end{array}$ & Método misto & IV \\
\hline E9 & $\begin{array}{l}\text { Saúde \& } \\
\text { Transformação } \\
\text { Social }\end{array}$ & $\begin{array}{l}\text { Moreira MA, } \\
\text { Carvalho CN19 }\end{array}$ & 2016 & $\begin{array}{c}\text { Atenção Integral à Saúde do } \\
\text { Homem: Estratégias utilizadas por } \\
\text { Enfermeiras(os) nas Unidades de Saúde } \\
\text { da Familia do interior da Bahia }\end{array}$ & Qualitativo & IV \\
\hline E10 & $\begin{array}{l}\text { Psicologia \& } \\
\text { Sociedade }\end{array}$ & $\begin{array}{l}\text { Pereira MCA, } \\
\text { Barros JPP20 }\end{array}$ & 2015 & $\begin{array}{l}\text { Públicos masculinos na estratégia de } \\
\text { saúde da família: estudo qualitativo em } \\
\text { Parnaíba - PI }\end{array}$ & Qualitativo & IV \\
\hline E11 & $\begin{array}{l}\text { Escola Anna } \\
\text { Nery Revista de } \\
\text { Enfermagem }\end{array}$ & $\begin{array}{l}\text { Cavalcanti JRD } \\
\quad \text { et al. }{ }^{8}\end{array}$ & 2014 & $\begin{array}{c}\text { Assistência integral à saúde do homem: } \\
\text { necessidades, obstáculos e estratégias } \\
\text { de enfrentamento }\end{array}$ & $\begin{array}{l}\text { Estudo } \\
\text { exploratório }\end{array}$ & IV \\
\hline E12 & $\begin{array}{l}\text { Escola Anna } \\
\text { Nery Revista de } \\
\text { Enfermagem }\end{array}$ & $\begin{array}{l}\text { Cordeiro SVL et } \\
\text { al. }^{21}\end{array}$ & 2014 & $\begin{array}{c}\text { Atenção básica à saúde masculina: } \\
\text { possibilidades e limites no atendimento } \\
\text { noturno }\end{array}$ & $\begin{array}{l}\text { Estudo } \\
\text { exploratorio }\end{array}$ & IV \\
\hline E13 & $\begin{array}{l}\text { Escola Anna } \\
\text { Nery Revista de } \\
\text { Enfermagem }\end{array}$ & $\begin{array}{l}\text { Albuquerque GA } \\
\text { et al. } .^{22}\end{array}$ & 2014 & $\begin{array}{l}\text { O homem na atenção básica: percepções } \\
\text { de enfermeiros sobre as implicações do } \\
\text { gênero na saúde }\end{array}$ & Qualitativo & IV \\
\hline E14 & $\begin{array}{l}\text { Escola Anna } \\
\text { Nery Revista de } \\
\text { Enfermagem }\end{array}$ & $\begin{array}{l}\text { Pereira } L P, \text { Nery } \\
\qquad A A^{23}\end{array}$ & 2014 & $\begin{array}{c}\text { Planejamento, gestão e ações à saúde } \\
\text { do homem na estratégia de saúde da } \\
\text { familia }\end{array}$ & Qualitativo & IV \\
\hline \multicolumn{7}{|c|}{ Elaboração: Bacelar GS, Aguiar RS, 2020.} \\
\hline
\end{tabular}

três (21,4\%), 2019, 2018 e 2016 com dois em cada ano (14,3\% em cada ano) e 2015 com um artigo $(7,1 \%)$. O desenho qualitativo foi o que mais prevaleceu dentre as pesquisas (seis artigos, 42,8\%). Sobre o nível de evidência dos artigos, todos (100\%) são de nível IV.

A análise temática dos resultados dos artigos permitiu a organização em duas categorias temáticas principais: 1) Estratégias utilizadas para inclusão do homem nas ações de saúde na APS e os fatores li- mitantes; e 2) Oportunidades de melhorias para inclusão do homem na APS.

\section{Estratégias utilizadas para inclusão do homem nas ações de saúde da APS e os fatores limitantes}

De maneira geral, os artigos trouxeram aspectos identificados e/ou executados pelos profissionais de saúde e gestores como necessários para inclusão do homem nas ações de saúde. Desse modo, o estabelecimento do vínculo entre o homem e os ser- viços da APS, a ampliação nos horários de atendimento e resolutividade das necessidades foram aspectos destacados pelos artigos E1, E3, E7.1,4,14

$\mathrm{O}$ estudo E1 apontou como estratégia de aproximação e coleta de dados dos sujeitos a visita domiciliar, acompanhada por um agente comunitário de saúde (ACS) responsável pela microárea da Estratégia Saúde da Família (ESF). ${ }^{4}$ A humanização foi um ponto destacado no estudo E11 entre as estratégias utilizadas para 
a inclusão do homem nas ações de saúde por favorecer a relação entre profissionais e usuários e facilitar a adesão aos serviços de saúde. ${ }^{8}$

$\mathrm{Na}$ percepção dos enfermeiros, identificou-se no estudo E8 que os homens valorizam o atendimento rápido e em menor tempo, reduzindo assim a espera no serviço de saúde. Ademais, os profissionais utilizam a oferta de preservativos para favorecer a participação masculina nas UBS. Adicionalmente, o deslocamento dos profissionais de saúde para o atendimento aos homens em seus espaços de trabalho, adaptando linguagens e materiais foram outras estratégias utilizadas. ${ }^{18}$

Os estudos E11 e E13 abordaram a acessibilidade através da ampliação dos horários de funcionamento da UBS e a resolutividade das necessidades. ${ }^{8,22}$ No que se refere as estratégias de inclusão, é valorizada no estudo E12 as palestras e campanhas educativas que valorizam as trocas interpessoais de conhecimento uma vez que proporcionam a interação da comunidade com os serviços de saúde e são capazes de mobilizar e motivá-los para um processo de mudança. ${ }^{21}$

Adicionalmente, os estudos E6 e E13 mostraram que algumas UBS desenvolveram estratégias de capacitação dos profissionais de saúde para atuarem frente à PNAISH e para consolidarem a construção de conhecimentos sobre a relação gênero e saúde. ${ }^{17,22}$ Ademais, o estudo E8 demonstrou a inclusão dos homens durante o pré-natal para possibilitar a realização de exames clínicos, planejamento reprodutivo e ações preventivas para a busca ativa de homens. ${ }^{18}$

A relação entre profissional e usuário foi um aspecto destacado no estudo E7 onde o vínculo entre o homem e o profissional de saúde é determinante para que este construa o sentimento de pertencimento àquele serviço. Ações ágeis e resolutivas, humanização no atendimento, acolhimento, vínculo entre o profissional e o usuário são estratégias importantes para a adesão dos homens aos serviços de saúde. ${ }^{1}$

A APS é vista como a porta de entrada preferencial dos usuários no Sistema Úni-

co de Saúde (SUS) devido a possibilidade de acompanhamento ao longo da vida e pela integralidade do cuidado, mas de acordo com os estudos E1 e E2 foi possível identificar algumas dificuldades de horários quando questionada a necessidade do homem ter que deixar o trabalho ou a escola para ir ao serviço de saúde, baixa resolutividade, tempo de espera maior que 30 minutos, dificuldades de obter conselhos por telefone sobre sua saúde e de comunicação quando a UBS está fechada. Para al-

Na percepção dos enfermeiros, identificou-se no estudo E8 que os homens valorizam o atendimento rápido e em menor tempo, reduzindo assim a espera no serviço

de saúde. Ademais, os profissionais utilizam a oferta de preservativos para favorecer a participação masculina nas UBS. guns usuários, a busca pelo serviço é considerada uma expressão de fragilidade e eles têm vergonha de procurar o serviço. ${ }^{4.5}$

Outros fatores limitantes identificados nos artigos E4, E7 e E9 foi a ausência de doenças, o medo de descobrir doença grave, a falta de acolhimento por parte dos profissionais de saúde, ausência de ações de promoção e de prevenção que trabalhe as singularidades do homem, deficiência dos serviços de saúde na oferta de recursos materiais, na marcação de exames e na falta de educação permanente para profissionais de saúde. ${ }^{1,15,19}$

Sobre a atuação dos enfermeiros, verificou-se no estudo E7 o desconhecimento da PNAISH e das práticas de cuidados específicas que devem ser ofertadas ao público masculino. Observou-se que alguns profissionais têm preconceito no sentido de ver o homem em busca de atendimento, principalmente no que se refere às questôes de prevenção e promoção da saúde. ${ }^{1}$

Adicionalmente, o estudo E12 demonstrou déficit de instrumentalização profissional no contexto da saúde do homem para a garantia de assistência qualificada quanto ao atendimento de suas necessidades de saúde ${ }^{21}$, além da valorização de práticas curativas e o não reconhecimento sobre a importância e a necessidade de ações de prevenção ou promoção à saúde de acordo com o estudo E13.22

\section{Oportunidade de melhorias para in- clusão do homem na APS}

Observou-se que existem poucas estratégias na APS para a inclusão do homem. Pensando nisso, os estudos E1, E2, E3 e E10 mostraram possíveis oportunidades de melhorias como organizar os serviços para melhor acolher a população masculina e responder positivamente às suas necessidades de saúde. Assim, destaca-se a resolutividade e a capacidade de vinculação do serviço com o usuário, bem como a resolução dos problemas, pois esses aspectos são imprescindíveis para efetivação enquanto contato e porta de entrada para os demais níveis de atenção. Além disso, melhorar a articulação, por parte dos profissionais e gestores, das políticas de saúde 
que tem o homem como protagonista, para fomentar ações que reconheçam as especificidades desse segmento populacional, vislumbrando a integralidade da assistência a essa população e implementar ações de educação em saúde que ajudem a aumentar as necessidades de cuidados masculinos. ${ }^{4-5,14,20}$

Adicionalmente, no estudo E3 fala sobre a realização de ações no horário noturno e/ou extensão dos horários de funcionamento, abertura de agendas específicas para $\mathrm{o}$ atendimento de homens. ${ }^{14}$ Ademais, identificou-se no estudo E5 outras oportunidades de melhorias como a flexibilização dos horários de atendimento, bem como a inclusão de atividades educativas específicas para o público masculino. ${ }^{16}$

Outras oportunidades em destaque apresentadas nos estudos E7, E8 e E9 foi a respeito da criação de novas ações para o cuidado com o público masculino em todas as faixas etárias, ampliação dos espaços de discussão entre o homem e o serviço de saúde, potencializar as oportunidades para realização de exames clínicos de rotina, valorizar a paternidade na estratégia do pré-natal e incluir os homens no planejamento familiar, investimentos e uma atenção em rede de modo que o âmbito local possa priorizar e executar estratégias em saúde de acordo com o perfil epidemiológico e de suas demandas populacionais com maior participação dos homens e implantação de serviços de referência, o que facilitaria a adesão dos mesmos às ações de saúde. ${ }^{1,18-19}$

Assim, é necessário que os profissionais de saúde se capacitem, problematizem a realidade de cada UBS e, juntamente com os gestores, vislumbrem e operacionalizem estratégias inclusivas de atendimento para o estudo E11. ${ }^{8}$

Os estudos E13 e E14 demonstraram que estabelecer parcerias com outros setores e instituições nas quais a população masculina está inserida é importante para promover um maior incentivo aos cuidados com a saúde e uma maior demanda pelos serviços da APS. Ainda, é importante pensar e discutir maneiras de inserir os profissionais de saúde nos ambientes onde a população masculina se concentra rotineiramente, como em locais de trabalho, ou ocasionalmente, em feiras e eventos. ${ }^{22-23}$

\section{Os estudos}

E13 e E14

demonstraram que

estabelecer parcerias

com outros setores

e instituições nas

quais a população

masculina

está inserida é

importante para

promover um

maior incentivo

aos cuidados com a

saúde e uma maior

demanda pelos

serviços da APS.

\section{DISCUSSÃO}

A APS é considerada a porta de entrada para os outros níveis de atenção ao cuidado. Desse modo, garantir a acessibilidade e o acolhimento ao homem é fundamental, bem como dispor de uma rede de serviços organizada para acolher a população masculina e apresentar uma resposta positiva as suas demandas de saúde. ${ }^{4}$

Diante disso, melhorar a articulação por parte dos profissionais e gestores, agregar ao modelo biomédico novas possibilidades de ações e serviços, praticar ações de educação em saúde que contribuem para o aumento da procura dos homens pelos atendimentos são necessárias para melhorar as estratégias de envolvimento dos homens nos serviços da APS. ${ }^{4-5}$

Ademais, para atender às peculiaridades da população masculina, é necessário que os profissionais de saúde se capacitem, problematizem a realidade de cada UBS e, juntamente com os gestores, vislumbrem e operacionalizem estratégias inclusivas de atendimento. Para isso, compreender mais a saúde do homem, sobretudo no que se diz a respeito do acesso e a utilização dos serviços de saúde, e, a partir de então, analisar e planejar ações que atendam às demandas desse grupo. ${ }^{8}$

Somando a esta necessidade, tem-se a identificação de atenção para a necessidade de investimento em melhorias na porta de entrada do SUS, por meio de qualificação dos profissionais e das ações de acolhimento aos homens e ressaltam a importância da construção de novos arranjos institucionais e gerenciais que contribuam para uma maior adesão masculina a esses espaços. ${ }^{4,14}$

Diante disso, é primordial a criação de estratégias específicas na APS direcionadas aos homens em idade adulta, especialmente, no que diz respeito à prevenção de agravos e à promoção de sua saúde ${ }^{8} \mathrm{e}$ a ampliação do diálogo em parcerias com as universidades, profissionais e gestores a fim de promover a valorização da contextualização crítica e histórica das nuances da atenção à saúde do homem. ${ }^{23}$

Portanto, organizar os serviços da APS para acolher a população e apresentar uma resposta positiva às suas demandas de saúde e, ainda agregar novas possibilidades de ações e programas, como arcabouço de eixos estruturantes das tecnologias cuidativas e sob pressupostos que reiterem a filosofia do SUS. ${ }^{4-5}$ 


\section{artigo}

Bacelar, G.S.; Aguiar, R.S.

Estratégias utilizadas na atenção primária para inclusão do homem nas ações de saúde: uma revisão integrativa

\section{CONCLUSÃO}

Identificou-se a existência de fragilidades na atenção à saúde do homem na APS que impactam diretamente no acesso dos homens aos serviços, a saber: ausência de ações promoção e prevenção a saúde, déficit de investimento e desconhecimento da
PNAISH. Diante disso, estes fatores acabam dificultando a realização de estratégias locais para a atenção integral à saúde do homem nos serviços da APS.

Paralelo a isso, foi identificado como aspectos positivos para inclusão do homem nas ações de saúde a visita domiciliar através do ACS, a realização de práticas dialogadas com a realização de palestras e campanhas educativas e o atendimento noturno a população masculina.

Portanto, torna-se necessário aperfeiçoar as ações voltadas para a população masculina nos serviços da APS para que haja o estímulo dos homens a adotarem a rotina de cuidado de sua saúde.

\section{REFERÊNCIAS}

1. Daher DV, Domingues PS, Gomes AMT, Nolasco MFS. A construção do vínculo entre o homem e o serviço de atenção básica de saúde. Rev Cuba enferm [Internet]. 2017 [Acesso em 1 nov 20]; 33(1):111-20.

2. Oliveira VB, Aguiar RS. Conhecimento da política de saúde do homem e a relação com a atenção à saúde. Saúde Coletiva (Barueri) [Internet]. 2020 [Acesso em 20 mar 21];10(55):2985-3002.

3. Balica LO, Aguiar RS. Percepções paternas no acompanhamento do pré-natal. Rev Aten Saúde [Internet]. 2019 [Acesso em 20 mar 21];17(61):114-126.

4. Alves ADN, Coura AS, França ISX, Magalhães IMO, Rocha MA, Araújo RS. Acesso de primeiro contato na atenção primária: uma avaliação pela população masculina. Rev Bras Epidemiol [Internet]. 2019 [Acesso em 1 nov 20];23:1-14.

5. Batista BD, Andrade ME, Gadelha MMT, Silva JMA, Fernandes PKRS, Fernandes MC. Discurso de homens sobre o acesso à saúde na atenção básica. Rev Baiana Enferm [Internet]. 2019 [Acesso em 1 nov 20];33:e29268.

6. Lima CS, Aguiar RS. Acesso dos homens aos serviços de atenção primária à saúde: uma revisão integrativa. Research, Society and Development [Internet]. 2020 [Acesso em 20 mar 21];9(4):e157943027.

7. Aguiar RS, Santana DC, Santana PC. A percepção do enfermeiro da estratégia saúde da família sobre a saúde do homem. Rev Enferm Cent 0 Min [Internet]. 2015 [Acesso em 20 mar 21];5(3):1844-1854.

8. Cavalcanti JRD, Ferreira JA, Henriques AHB, Morais GSN, Trigueiro JVS, Torquato IMB. Assistencia integral a saúde do homem: necessidades, obstaculos e estrategias de enfrentamento. Esc Anna Nery Rev Enferm [Internet]. 2014 [Acesso em 1 nov 20];18(4):628-34.

9. Lima RC, Aguiar RS. Experiência paterna com o recém-nascido a partir das orientações de enfermagem. Rev Cereus [Internet]. 2020 [Acesso em 20 mar 21];12(1):193-202.

10. Pluye P, Hong QN. Combining the power of stories and the power of numbers: mixed methods research and mixed studies reviews. Annu Rev Public Health [Internet]. 2014 [Acesso em 1 nov 20];35:29-45.

11. Donato $H$, Donato $M$. Stages for undertaking a systematic review. Acta Medica Portuguesa [Internet]. 2019 [Acesso em 1 nov 20];32(3):227-235.
12. OCEBM Levels of Evidence Working Group. The Oxford 2011 Levels of Evidence [Internet]. Oxford: Oxford Centre for Evidence-Based Medicine; 2011 [Acesso em 1 nov 20].

13. Braun V, Clarke V. Using thematic analysis in psychology. Qual Res Psychol [Internet]. 2006 [Acesso em 1 nov 20]; 3(2):77-101.

14. Bacelar AYS, Coni DGL, Santos DV, Sousa AR. Homens na unidade de saúde da família. Rev Enferm UFPE on line [Internet]. 2018 [Acesso em 1 nov 20];12(9):2507-13.

15. Barbosa YO, Menezes LPL, Santos AD, Cunha JO, Santos JMDJ, Menezes AF et al. Acesso dos homens aos serviços de atenção primária à saúde. Rev Enferm UFPE on line [Internet]. 2018 [Acesso em 1 nov 20];12(11):2897-905.

16. Silva NA, Silva SA, Silva ARV, Araújo TME, Rebouças CBA, Nogueira LT. A avaliação da atenção primária a saúde na perspectiva da população masculina. Rev Bras Enferm [Internet] 2018 [Acesso em 1 nov 20];71(2):255-63.

17. Martins AM, Modena CM. Acesso da população masculina e utilização dos serviços de atenção primária à saúde em belo horizonte - MG. Rev APS [Internet]. 2017 [Acesso em 1 nov 20];20(4):482-92.

18. Moreira MCN, Gomes R, Ribeiro CR. E agora o homem vem?! Estratégias de atenção à saúde dos homens. Cad Saude Publica [Internet]. 2016 [Acesso em 1 nov 20];32(4):e00060015.

19. Moreira MA, Carvalho CN. Atenção Integral à Saúde do Homem: Estratégias utilizadas por enfermeiras(os) nas Unidades de Saúde da Família do interior da Bahia. Sau \& Transf Soc [Internet]. 2016 [Acesso em 1 nov 20];7(3):121-32.

20. Pereira MCA, Barros JPP. Públicos masculinos na estratégia de saúde da família: Estudo qualitativo em Parnaíba-PI. Psicol Soc [Internet]. 2015 [Acesso em 1 nov 20];27(3):587-98.

21. Cordeiro SVL, Fontes WD, Fonsêca RLS, Barboza TM, Cordeiro CA. Atenção básica à saúde masculina: possibilidades e limites no atendimento noturno. Esc Anna Nery Rev Enferm [Internet]. 2014 [Acesso em 1 nov 20];18(4):644-9.

22. Albuquerque GA, Leite MF, Belém JM, Nunes JFC, Oliveira MA, Adami F. O homem na atenção básica: percepções de enfermeiros sobre as implicações do gênero na saúde. Esc Anna Nery Rev Enferm [Internet]. 2014 [Acesso em 1 nov 20];18(4):607-614.

23. Pereira LP, Nery AA. Planejamento, gestão e ações à saúde do homem na estratégia de saúde da família. Esc Anna Nery Rev Enferm [Internet]. 2014 [Acesso em 1 nov 20];18(4):635-43. 